\title{
Electron Microscopy and X-ray Diffraction Study of AlN Layers
}

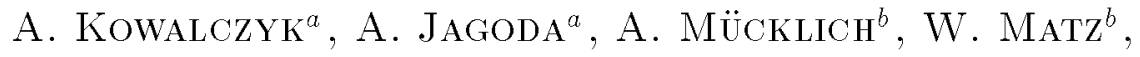 \\ M. PawŁowska ${ }^{a}$, R. RatajczaK ${ }^{c}$ AND A. Turos ${ }^{a, c}$ \\ ${ }^{a}$ Institute of Electronic Materials Technology \\ Wólczyńska 133, 01-919 Warsaw, Poland \\ ${ }^{b}$ Forschungszentrum Rossendorf, IIM, POB 510119, 01314 Dresden, Germany \\ ${ }^{c}$ Soltan Institute for Nuclear Studies, Hoża 69, 00-681 Warsaw, Poland
}

AIN nanocrystalline layers and superstructures are used in the modern optoelectronic technology as reflecting mirrors in semiconductor lasers. In the present work the properties of AIN films prepared by sputtering methods from an AlN target in reactive $\mathrm{Ar}+\mathrm{N}$ plasma were investigated. The characterisation was performed with HRTEM, SEM, glancing angle XRD and RBS methods. The present measurements confirmed the polycrystalline structure of AlN layers and enabled the evaluation of their grain size. The roughness and thickness of the layers were additionally determined by ellipsometric and profilometric measurements.

PACS numbers: 52.77.Dq

\section{Introduction}

Aluminium nitride, which is a III-V family compound, is of the hexagonal structure with the space group $P 6_{3} m c$ (No. 186). The growth and structural perfection of AlN thin layers was recently described by Takikawa et al. [1], Karmann et al. [2], Xiao-Hong $\mathrm{Xu}$ et al. [3] and Wang et al. [4].

The presently studied AIN layers were grown by means of the AIN target sputtering in reactive $\mathrm{Ar}+\mathrm{N}$ plasma. AIN layers of thickness from 2 to $5 \mu \mathrm{m}$ have been deposited on the Si substrate. Multiple AIN-Si layers, each of $100 \mathrm{~nm}$ thickness, were also studied. The samples were characterized using various methods. The initial selection of samples with appropriate refraction index $(n=2 \pm 0.2)$ was made by means of ellipsometry. The morphology of the surface was examined with scanning electron microscopy (SEM), while the structural analysis was 
performed using transmission electron microscopy (TEM) and X-ray diffraction (XRD) techniques. The multi-layer structure was additionally analyzed using the Rutherford backscattering spectrometry (RBS) method. The preliminary results of investigation of similar AlN layers grown at the Institute of Electronic Materials Technology were described in $[5,6]$.

\section{Experimental}

The AlN layers were deposited using SCM 450 Alcatel sputtering facility equipped with RF-600W, DC-2kW and RF-300W generators in the inverted target-substrate configuration. The vacuum in the reaction chamber was $10^{-7} \mathrm{mbar}$. Layers were deposited by sputtering from an AlN target in reactive $\mathrm{Ar}+\mathrm{N}$ plasma. Before the deposition of layers the substrates were cleaned by plasma etching at the maximum rate of $0.2 \mathrm{~nm} / \mathrm{min}$.

Three types of samples were studied: (i) AlN layers of $2.5 \mu \mathrm{m}$ thickness on Si substrates, (ii) AlN layers of $4.3 \mu \mathrm{m}$ thickness on Si substrates, and (iii) a multi-layer structures $\mathrm{Si}(46.1 \mathrm{~nm}) / \mathrm{AlN}(79.9 \mathrm{~nm}) / \mathrm{Si}(41 \mathrm{~nm}) / \mathrm{AlN}(79.3 \mathrm{~nm})$ on Si substrates.

XRD studies were performed with the Siemens D5000 diffractometer equipped with the Göbel-mirror and a thin film attachment. The grazing incidence technique with incidence angles of $2^{\circ}$ and $4^{\circ}$ was applied and $\mathrm{Cu} K_{\alpha}$ radiation was used.

Specimens for TEM investigation were prepared in the form of a cross-section, where the (112) planes of the Si substrate were normal to the specimen. The SEM observations were performed with an OPTON Digital Scanning Microscope DSM 950.

For RBS studies the beam of ${ }^{4} \mathrm{He}^{+}$ions with the energy of $1.7 \mathrm{MeV}$ was used. The backscaterred ions were recorded at $170^{\circ}$. The evaluation of the layer thickness was performed using RUMP program [7].

\section{Results}

The typical glancing angle X-ray diffraction patterns for an incidence angle of $4^{\circ}$ are shown in Fig. 1. Two types of structure are indicated in Fig. 1: hexagonal $\mathrm{AlN}$ and cubic Si. All observed diffraction peaks belong to the hexagonal AlN phase (space group No. 186).

The lattice constants of AlN had to be modified in order to explain the recorded peak positions. The actual values (database values are given in brackets) are: $a=0.31217 \mathrm{~nm}(0.31114)$ and $c=0.49979 \mathrm{~nm}(0.49792)$. That means that the lattice of AlN crystallites is expanded in both directions. From the peak intensities one can deduce a preferred orientation of the basal plane (002) documented by the very high peak at $36^{\circ}$. This effect becomes more pronounced with increasing thickness of AlN layers (from $2.5 \mu \mathrm{m}$ to $4.3 \mu \mathrm{m}$ ). Since XRD measurements were 


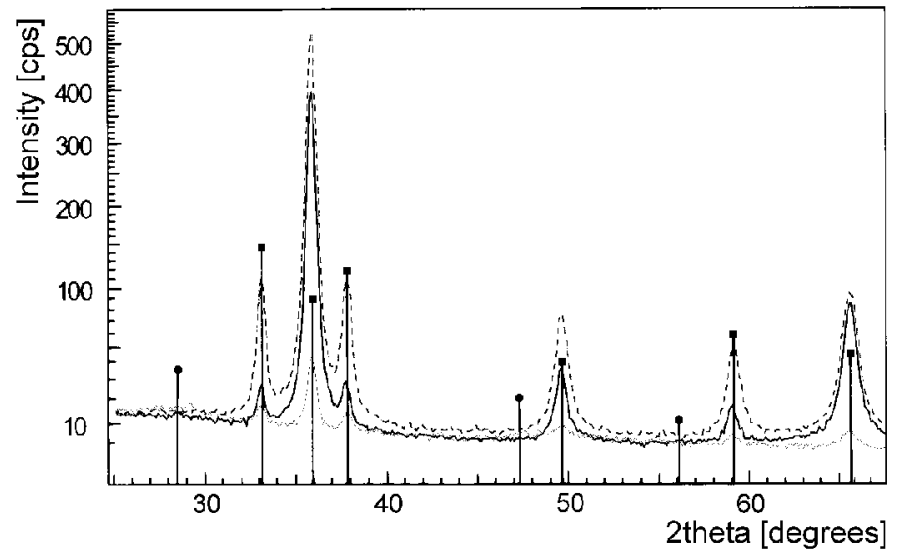

Fig. 1. X-ray diffraction patterns obtained using the grazing incidence technique for the AIN/Si samples. Dashed line: AIN $2.5 \mu \mathrm{m}$ on Si substrate, thick line: AlN $4.3 \mu \mathrm{m}$ on Si substrate, thin line: a multi-layer structure $(\mathrm{Si}-\mathrm{AlN}) \times 2$ on $\mathrm{Si}$ substrate, $\square$ reference position of hexagonal AlN reflection, • reference position of Si reflection.

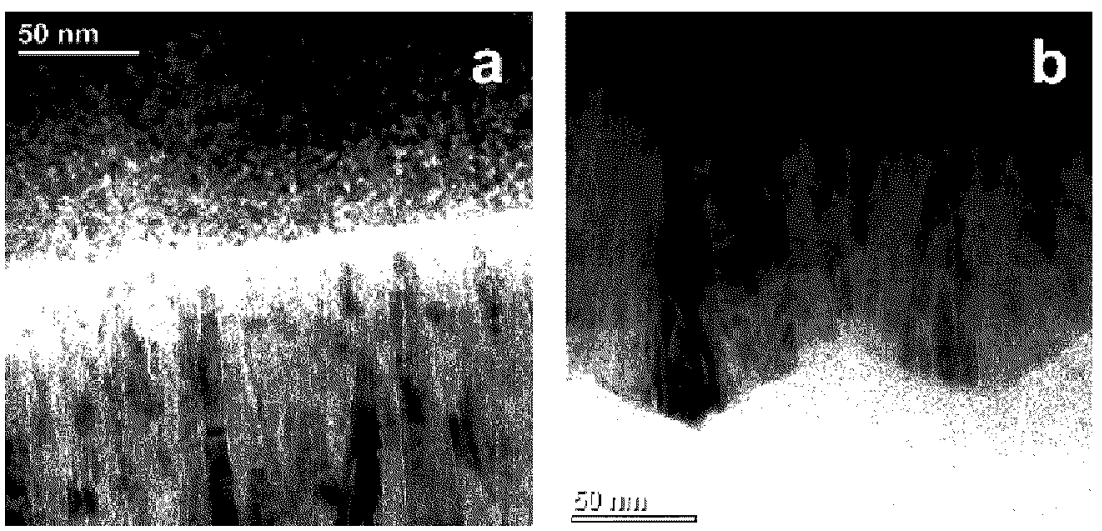

Fig. 2. TEM micrographs of AIN layers deposited on Si substrate: (a) AlN-Si interface, (b) surface roughness.

performed only at the grazing incidence, only a qualitative discussion of the preferred orientation was possible. However, a similar effect was found for the nearest basal plane reflection (103) at 65.8 ${ }^{\circ}$. In the multi-layer sample only AlN reflections are found, while the Si layers are apparently amorphous.

The estimation of crystallite size from peak FWHMs using the Scherrer formula for the thick layers gives contradictory results. The broadening of each peak was estimated separately. For the $2.5 \mu \mathrm{m}$ layer one can estimate the coherence sizes of about $25 \mathrm{~nm}$ from the (100) and (101) reflections (which are nearly perpendicular to the basal plane). For the (002) and (103) reflections, which are of 
more basal plane orientation, the result of estimation is 13 to $8 \mathrm{~nm}$. The other reflections provided the values between these two limits. For the $4.2 \mu \mathrm{m}$ layer a similar tendency was found, but the absolute values were reduced by about $25 \%$. The reason of that may be the strain in the thick layers. Moreover, the deposition was performed stepwise with identical intervals at several hours. As a consequence a layered structure was formed with different crystallite sizes. For the multi-layer a more uniform crystallite size at about $15 \mathrm{~nm}$ from all reflections was found.

The typical TEM images for $2.5 \mu \mathrm{m}$ AlN layer are shown in Fig. 2. The first micrograph shows the interface region, while another one shows the roughness of the surface. In the high-resolution TEM images of single crystalline grains were observed. Their sizes were approximately the same as deduced from the XRD measurements.

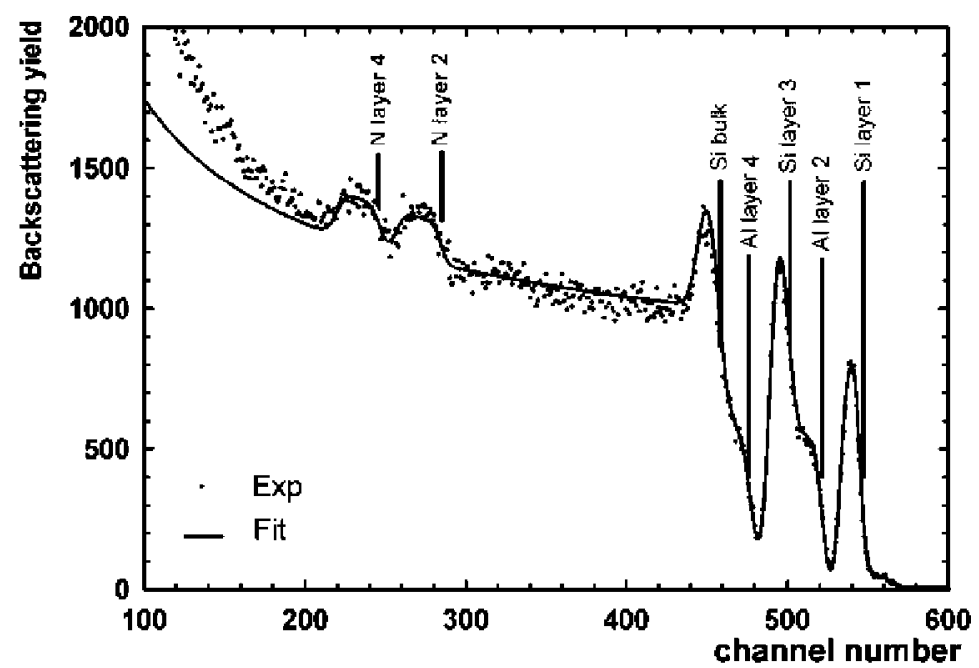

Fig. 3. RBS experimental spectrum in random geometry (dots) and calculated spectrum (solid line) for a multi-layer structure (Si/AlN/Si/AlN/S).

The RBS technique enabled identification and evaluation of the concentration of subsequent atomic components as well as the determination of their depth distributions. The experimental RBS spectrum for multi-layers Si/AlN/Si/AlN on Si substrate and a theoretical fit are presented in Fig. 3. The multi layer structure as deducted from the RBS analysis are shown in Fig. 4.

\section{Conclusions}

The XRD measurements performed with grazing incidence technique confirmed that the investigated samples were of the hexagonal structure with lattice 


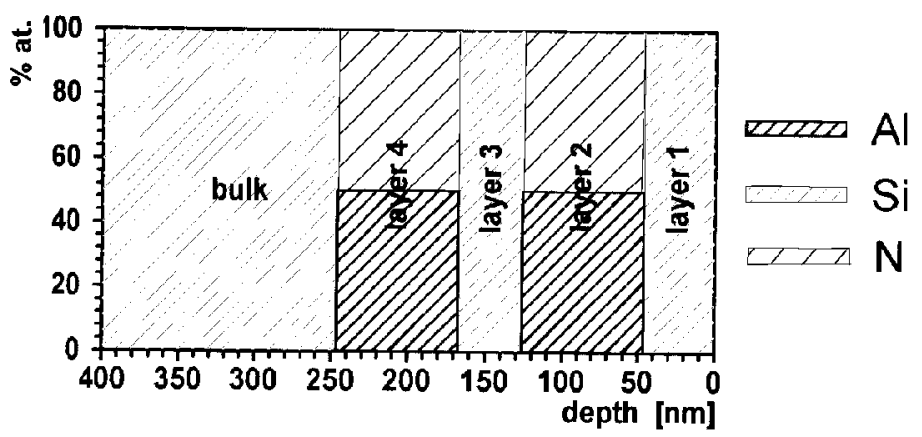

Fig. 4. Si/AlN/Si/AlN/Si multi-layer structure as deducted from the RBS analysis.

parameters slightly greater than the database values. The preferential grain orientation along (002) planes was found. The diffraction patterns revealed the existence of well-developed crystallites. The size of crystallites increased with increasing layers thickness. In all cases the morphology of the AlN-layers is characterized by columnar structures. According to TEM analysis, the surface roughness is limited by the size of crystal grains. The TEM images provided similar evaluation of the grain size as XRD measurements. The sharp interfaces between a layer and the substrate and between the layers in the multi-layer sample were also revealed. RBS experiments provided important information on the real thickness of subsequent layers in the AIN-Si multi-layer sample.

\section{References}

[1] H. Takikawa, K. Kimura, R. Miyana, T. Sakakibara, A. Bendavid, P.J. Martin, A. Matsumuro, K. Tsutsumi, Thin Solid Films 386, 276 (2001).

[2] S. Karmann, H.P.D. Schenk, U. Kaiser, A. Fissel, Wo. Richter, Mater. Sci. Eng. $B$ 50, 228 (1997).

[3] Xiao-Hong Xu, Hai-Shun Wu, Cong-Jie Zhang, Zhi-Hao Jin, Thin Solid Films 388, $62(2001)$.

[4] X.D. Wang, U. Mazur, J.T. Dickson, Thin Solids Film 240, 45 (1994).

[5] A. Jagoda, L. Dobrzański, M. Możdżonek, in: VII Konferencja Naukowa "Technologia Elektronowa”, ELTE 2000, Polanica Zdrój (Poland) 2000, p. 234 (in Polish).

[6] A. Jagoda, L. Dobrzański, K. Dąbrowska, K. Przyborowska, in: VII Konferencja Naukowa "Technologia Elektronowa", ELTE 2000, Polanica Zdrój (Poland) 2000, p. 717 (in Polish).

[7] J.W. Martin, D.D. Cohen, N. Dytlewski, D.B. Garton, H.J. Whitlow, G.J. Russell, Nucl. Instrum. Methods Phys. Res. B 94, 277 (1994). 